University of Warwick institutional repository: http://go.warwick.ac.uk/wrap This paper is made available online in accordance with publisher policies. Please scroll down to view the document itself. Please refer to the repository record for this item and our policy information available from the repository home page for further information.

To see the final version of this paper please visit the publisher's website. Access to the published version may require a subscription.

Author(s): M. A. Sadeghzadeh

Article Title: Top-gating of $\mathrm{p}-\mathrm{Si} / \mathrm{SiGe} / \mathrm{Si}$ inverted modulation-doped structures

Year of publication: 2000

Link to published version: http://dx.doi.org/10.1063/1.125750

Publisher statement: None 


\title{
Top-gating of $p$-Si/SiGe/Si inverted modulation-doped structures
}

\author{
M. A. Sadeghzadeh ${ }^{\text {a) }}$ \\ Department of Physics, University of Warwick, Coventry, CV4 7AL, United Kingdom
}

(Received 3 June 1999; accepted for publication 13 November 1999)

Low-temperature electrical properties of two-dimensional hole gases (2-DHGs) in $\mathrm{Si} / \mathrm{Si}_{0.8} \mathrm{Ge}_{0.2} / \mathrm{Si}$ inverted modulation-doped structures have been investigated at different hole densities using a metal semiconductor gate sputtered on top of these structures. The 2-DHG which is supplied to the inverted interface of $\mathrm{Si} / \mathrm{SiGe} / \mathrm{Si}$ quantum well by a $\mathrm{Si}$ boron-doped layer spatially grown beneath the alloy, was controlled in the range of $1.5-7.8 \times 10^{11} \mathrm{~cm}^{-2}$ hole density by biasing the top gate. With increasing 2-DHG sheet density, the hole wave function of these structures expands and moves away from inverted interface, consequently the mobility enhances. These results may be understood theoretically by elaborating the role of interface charge, roughness, and alloy scattering mechanisms in limiting the mobility of holes at the inverted interface. (C) 2000 American Institute of Physics. [S0003-6951(00)03802-X]

Pseudomorphically grown $\mathrm{Si} / \mathrm{SiGe} / \mathrm{Si}$ structures are of great interest in physics and field-effect transistor (FET) applications. ${ }^{1-3}$ A quasi two-dimensional hole gas (2-DHG) can be formed in the compressively strained SiGe quantum well either by a metal-oxide-semiconductor (MOS) gate fabricated on top of these structures ${ }^{4}$ or by $p$-type (boron) remote doping before and/or after the SiGe channel corresponding to the inverted and/or normal modulation-doped (MD) configurations, respectively. ${ }^{5,6}$ Structures with a controllable hole density in the channel are useful in understanding the scattering mechanisms limiting mobility of holes confined near to normal ( $\mathrm{Si}$ on $\mathrm{SiGe}$ ) and inverted ( $\mathrm{SiGe}$ on $\mathrm{Si}$ ) interfaces of the $\mathrm{Si} / \mathrm{SiGe} / \mathrm{Si}$ quantum wells, so gating these structures allows one to change the hole sheet density systematically in a single device. MOS gating of undoped $\mathrm{Si} / \mathrm{SiGe} / \mathrm{Si}$ structures ${ }^{4}$ and back-gating of normal MD structures ${ }^{7,8}$ has been reported to study the transport properties of holes confined at the normal interface. Here we present the low-temperature transport properties of holes at the inverted interface of $\mathrm{Si} / \mathrm{Si}_{0.8} \mathrm{Ge}_{0.2} / \mathrm{Si}$ structures by means of a metal-semiconductor (Schottky) gate deposited on top of these structures and a Si boron-doped layer beneath the alloy.

The inverted MD structures used in this work were grown by solid-source molecular-beam epitaxy (MBE) on low doped ( $n$-type, $1-2 \Omega \mathrm{cm}$ ) Si (100) substrates and reported previously. ${ }^{5}$ These structures consisted of the following layer sequence: $200 \mathrm{~nm} \mathrm{Si} \mathrm{buffer,} 30 \mathrm{~nm} \mathrm{Si}$ boron-doped at $2 \times 10^{18} \mathrm{~cm}^{-3}, 10-20 \mathrm{~nm}$ Si spacer $\left(l_{s}\right), 17-20 \mathrm{~nm}$ nominally $\mathrm{Si}_{0.8} \mathrm{Ge}_{0.2}$ alloy $\left(l_{w}\right)$, and finally a $\mathrm{Si}$ cap layer $\left(l_{c}\right)$ of nominally 150 or $400 \mathrm{~nm}$ thickness.

A cross-sectional view of the devices fabricated in this work is shown in Fig. 1(a). The first step in device processing was to make electrical contact to the buried 2-DHG, by sputtering $\mathrm{Al}$ and annealing in ambient nitrogen. Then Ti/Al metal-semiconductor (Schottky) gate was sputtered on $\mathrm{Si}$ cap of the device channels. Standard Van der Pauw and Hall

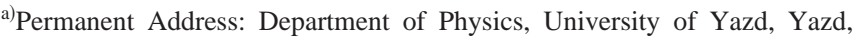
Iran; electronic mail: sadeghtaj@hotmail.com
}

bar devices (with a channel length of $3300 \mu \mathrm{m}$, a channel width of $315 \mu \mathrm{m}$, voltage probes $1260 \mu \mathrm{m}$ apart) were made by photolithography techniques and wet etching.

The valence band profile of these structures is shown in Fig. 1(b). At electrostatic equilibrium, the areal density of the ionized boron atoms in the depletion region of supply layer, and those segregated into the spacer layer, counterbalance the 2-DHG sheet density $n_{s}$ at the inverted interface, and the total cap-side charge density $n_{c}$. The cap-side charge density consists of metal-semiconductor charges, and depleted charges arising from background ( $n$-type) impurities in the cap layer. By applying a commensurate voltage to the gate $\left(V_{g}\right)$, an additional charge density is induced at the metal-semiconductor contact. As a consequence the electric field in the cap, alloy, and spacer alters and thereby an adjustment to the quantum well shape together with a shift of the valence band edge in the well relative to the Fermi energy, leads to modulation of the 2-DHG density from a measurement limited value $\left(\sim 1.5 \times 10^{11} \mathrm{~cm}^{-2}\right)$ up to maximum value (depends on the spacer layer thickness and alloy composition) correspond to the almost flat band situation in the cap.

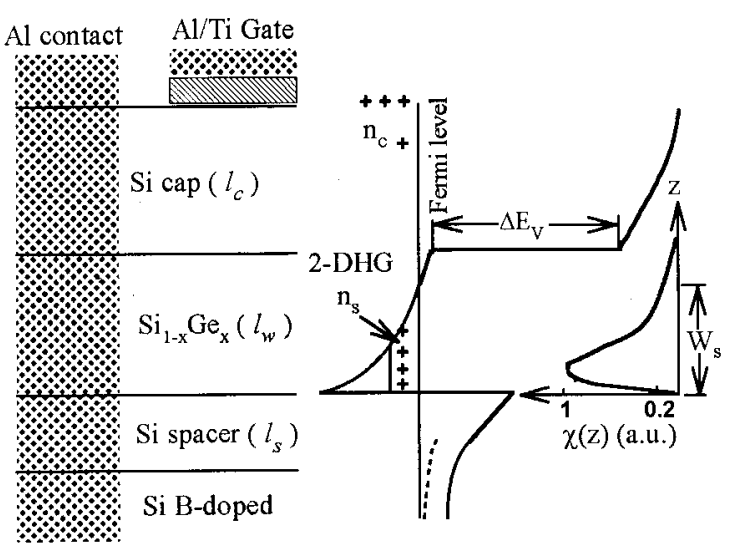

(a)

(b)

FIG. 1. (a) A cross-sectional view (not to scale) of the top-gated device fabricated in this work, and (b) the corresponding valence band profile in the structure. Also a schematic hole wave function $\chi(z)$ is shown in the inset. 
TABLE I. The structural parameters and mobility $\mu$ (at $4.2 \mathrm{~K}$ ) for the maximum hole density $n_{s}$ of top-gated structures under study.

\begin{tabular}{cccccc}
\hline \hline $\begin{array}{c}\text { Sample } \\
\text { i.d. }\end{array}$ & $\begin{array}{c}\mathrm{Ge} \% \\
(x)\end{array}$ & $\begin{array}{c}\text { Spacer } \\
l_{s} \\
(\mathrm{~nm})\end{array}$ & $\begin{array}{c}\text { Cap } \\
l_{c} \\
(\mathrm{~nm})\end{array}$ & $\begin{array}{c}\text { Maximum } \\
n_{s} \\
\times 10^{11} \mathrm{~cm}^{-2}\end{array}$ & $\begin{array}{c}\text { Hall mobility } \\
\mathrm{cm}^{2} \mathrm{~V}^{-1} \mathrm{~s}^{-1}\end{array}$ \\
\hline $\mathrm{A}$ & 17 & 20 & 480 & 4.2 & 5250 \\
$\mathrm{~B}$ & 20 & 20 & 150 & 5.2 & 4550 \\
$\mathrm{C}$ & 23 & 20 & 380 & 6.2 & 3950 \\
$\mathrm{D}$ & 20 & 10 & 420 & 7.8 & 3420 \\
\hline \hline
\end{tabular}

Resistivity and Hall effect measurements were carried out for a $500 \mathrm{nA}$ ac current using the lock-in amplifier method at temperatures down to $1.6 \mathrm{~K}$. The structural parameters and the Hall mobility $\mu$ (at $4.2 \mathrm{~K}$ ) for the maximum hole sheet density of top-gated inverted MD structures under study are listed in Table I for comparison. X-ray measurements showed that there was some deviation from the intended value $(x=0.20)$ in sample $\mathrm{A}(x=0.17)$ and $\mathrm{C}(x$ $=0.23$ ).

Despite the large gate area, the leakage current in the electrical measurement range was negligible $(20 \mathrm{pA})$ compared with the desired in-plan current along the channel and a typical measured leakage is shown in Fig. 2(a). The variations of the hole sheet density of these top-gated structures (at $4.2 \mathrm{~K}$ ) versus gate voltage are shown in Fig. 2(b). As seen, at $V_{g}=0$ the holes at the inverted interface are depleted by metal-semiconductor interface states to some extent. This effect was more pronounced in sample B which had a 150

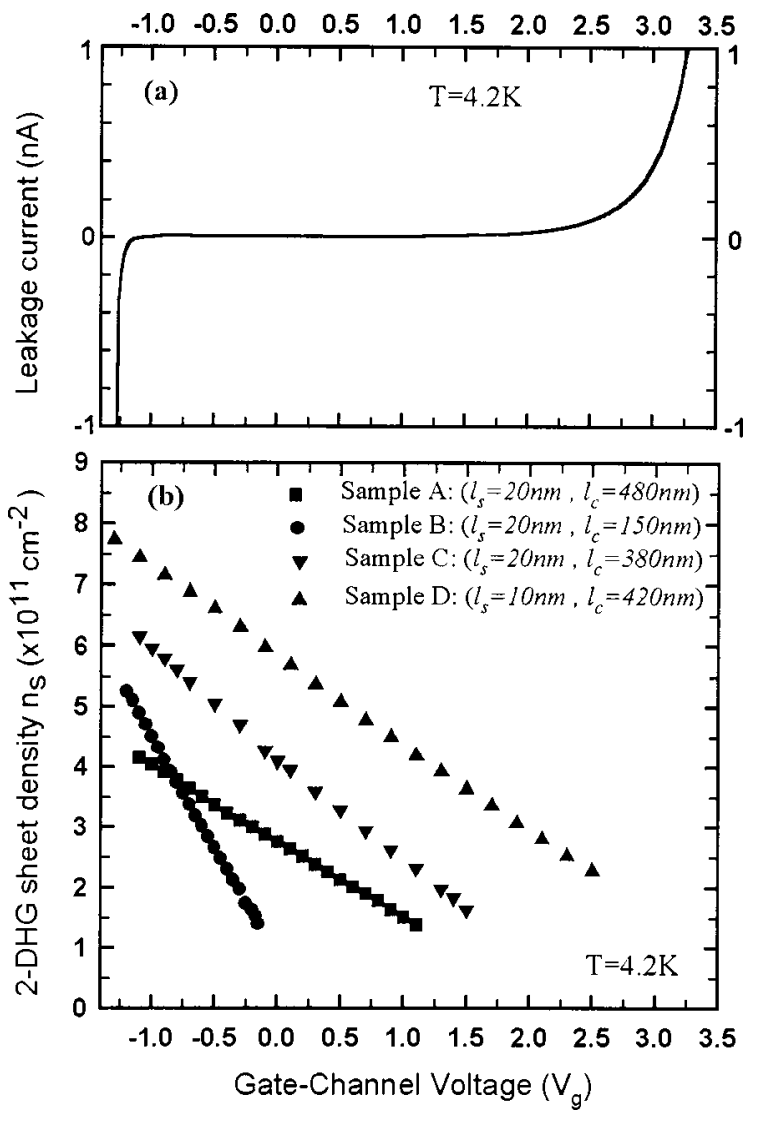

FIG. 2. The variations of (a) typical leakage current (for example B) and, (b) 2-DHG sheet density $n_{s}$ as a function of gate bias voltage for structures under study. Study, a self-consistent solution of Schrodinger's and Pois-
Downloaded 06 Jul 2009 to 137.205.202.8. Redistribution subject to AIP license or copyright; see http://apl.aip.org/apl/copyright.jsp
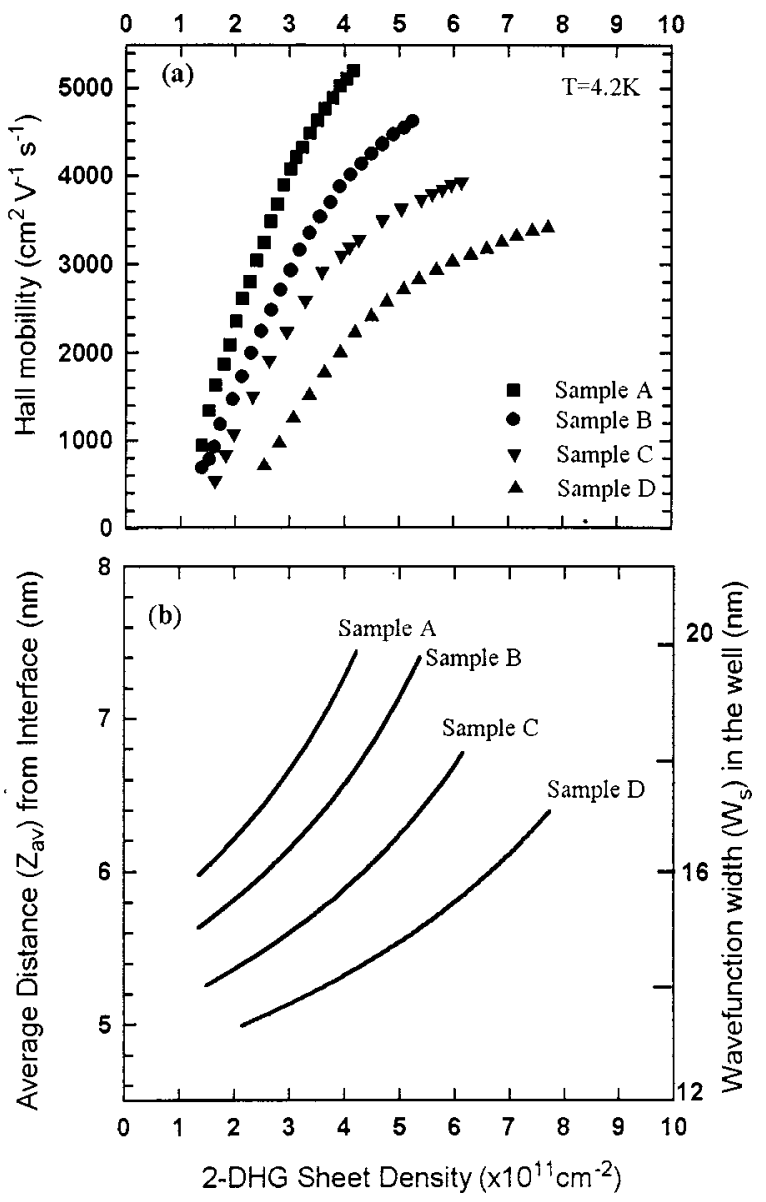

FIG. 3. Variations of (a) Hall mobility $\mu$ (measured at $4.2 \mathrm{~K}$ ), and (b) the calculated average distance $Z_{\text {av }}$ (left axis) and wave function width $W_{s}$ (right), as a function of hole density.

$\mathrm{nm} \mathrm{Si}$ cap. In the negative bias region, the metalsemiconductor interface charges are balanced with gateinduced charges, and with increasing negative bias, the 2-DHG sheet density $n_{s}$ increases and approaches to the maximum value which can be accommodated in the channel. The maximum hole sheet density attainable in each structure depends on the spacer layer thickness $l_{s}$, and the valence sition. The maximum hole sheet densities measured for these structures were higher than expected, but can be explained by a reduction of spacer layer thickness due to boron segregation effect. ${ }^{5}$ The slopes of linear changes of the hole sheet density with gate voltage for these structures are in agreement with $\epsilon \epsilon_{r} / e L$ relationship seen in all gated structures, where, $\epsilon$ and $\epsilon_{r}$ are respectively the permittivity of free space and dielectric constant of $\mathrm{Si}$, and $L$ is the distance between the metal gate and 2-DHG, and finally $e$ is the electron charge.

The variations of the Hall mobility $\mu$ (at $4.2 \mathrm{~K}$ ) with the hole sheet density for these structures are shown in Fig. 3(a). At low densities the mobility changes almost proportionally to the hole density but at high densities, its rate decreases.

The interesting feature of this kind of gating is the displacement of wave function from interface at higher densities which is in contrast to MOS gating structures but similar to the case of back-gating of normal MD structures. For this band discontinuity $\Delta E_{\mathrm{v}}$ which is proportion to alloy compo- 
son's equations was carried out for these structures taking 30 $\mathrm{meV}$ for boron binding energy, and 155, 180, and $210 \mathrm{meV}$ for valance band discontinuity of $17 \%, 20 \%$, and $23 \%$ alloy compositions, respectively. Moreover, it was assumed that the Ge has a rectangular profile in the well. Four sets of cap-side charge densities were calculated self-consistently for the corresponding measured hole densities in these structures. Ignoring the penetration of wave function in the barrier, the average distance of holes from the interface $Z_{\text {av }}$ were calculated by taking the Fang Howard wave function:

$$
\chi(z)=\left(b^{3} / 2\right)^{1 / 2} z \exp (-b z / 2),
$$

where $b$ is the variational parameter, which for the inverted structures can be written as:

$$
b=\left\{\frac{12 m^{*} e^{2}}{\hbar^{2} \epsilon \epsilon_{r}}\left[n_{c}+\frac{11}{32} n_{s}\right]\right\}^{1 / 3},
$$

where $m^{*}$ is the hole effective mass which an average value of $0.26 m_{0}$ was taken as extracted from Shubnikov-de Haas experiments in such structures. ${ }^{9}$ Since the 2 -DHG sheet density $n_{s}$ and cap-side charge density $n_{c}$ have different coefficients in Eq. (2), so the average distance $\left(Z_{\mathrm{av}}=3 / b\right)$ of the hole wave function from interface is sensitive to hole density $n_{s}$, regarding that the changes in the sum of $n_{s}$ and $n_{c}$ does not exceed more than $10 \%$ for the range of measured densities in each structure. These calculations can also evaluate the width of the wave function in the quantum well $W_{s}$ $=8 / \mathrm{b}$ [see inset to Fig. 1(b)]. The calculated average distance of holes from the inverted interface for these structures are shown in Fig. 3(b). As seen, with increasing hole sheet density, the wave function becomes wider and moves away from inverted interface. Moreover, at a fixed hole density, the wave function is closer to the interface for the higher Ge composition (samples B and C) or smaller spacer thickness (sample D).

The low mobility (at $4.2 \mathrm{~K}$ ) of holes confined at the $\mathrm{Si} / \mathrm{SiGe} / \mathrm{Si}$ structures has been a matter of controversy $4,6,10,11$ and interface charge, roughness, strain fluctuation, and alloy scattering potentials have been addressed for it. For example, in Ref. 4 it was argued that the mobility of holes at the normal interface measured in MOS gated structures is limited by interface charges at low densities, but switches to other scattering potentials at high densities $\left(n_{s}>5\right.$ $\times 10^{11} \mathrm{~cm}^{-2}$ ) while the others addressed just the alloy scattering for the case. Here, due to abnormal expansion and movement of the hole wave function with increasing hole density, there is a concomitant effect in decreasing of the efficiency of interface charge, roughness, and alloy scattering mechanisms. Despite this fact, the variations of Hall mobility with hole density (having downward curvatures) are rather similar to those in normal interface ${ }^{4,8}$ and this is not understandable. Regarding that, we did not observe any pronounced difference in the mobility of holes confined at normal and inverted interfaces of $\mathrm{Si} / \mathrm{Si}_{0.8} \mathrm{Ge}_{0.2} / \mathrm{Si}$ structures, ${ }^{5} \mathrm{a}$ theoretical calculation is needed to elaborate the role of these short range scattering mechanisms in limiting the mobility of holes at $\mathrm{Si} / \mathrm{SiGe} / \mathrm{Si}$ interfaces and explain these experimental results.

In conclusion, metal-semiconductor (Schottky) devices were fabricated on top of $\mathrm{Si} / \mathrm{Si}_{0.8} \mathrm{Ge}_{0.2} / \mathrm{Si}$ structures having a boron doped slab beneath the alloy, and with different spacer and Si cap layers. Low-temperature electrical properties of 2-DHGs near the inverted interfaces of these structures have been investigated for hole densities in the range of 1.5-7.8 $\times 10^{11} \mathrm{~cm}^{-2}$ in single devices through biasing the top-gate. The enhancement in mobility with increasing 2-DHG sheet density may be attributed to the abnormal expansion of hole wave function and its movement from inverted interface. These experimental results may be understood theoretically by elaborating the role of interface charge, roughness, and alloy scattering mechanisms in limiting the mobility of holes at the inverted interface of $\mathrm{Si} / \mathrm{SiGe} / \mathrm{Si}$ structures.

The author would like to thank E. H. C. Parker, T. E. Whall, O. A. Mironov, C. J. Emeleus (Glasgow University), P. J. Phillips, and C. P. Parry for useful discussions and sample supply.

${ }^{1}$ P. T. Coleridge, R. L. Williams, Y. Feng, and P. Zawadzki, Phys. Rev. B 56, 12764 (1997).

${ }^{2}$ T. E. Whall, Thin Solid Films 294, 160 (1997).

${ }^{3}$ S. Verdonckt-Vandebroek, E. F. Crabbe, B. S. Meyerson, D. L. Harame, P. J. Restle, J. M. C. Stork, and J. B. Johnson, IEEE ED 41, 90 (1994).

${ }^{4}$ R. J. P. Lander, M. J. Kearney, A. I. Horrell, E. H. C. Parker, P. J. Phillips, and T. E. Whall, Semicond. Sci. Technol. 12, 1064 (1997).

${ }^{5}$ M. A. Sadeghzadeh, C. P. Parry, P. J. Phillips, E. H. C. Parker, and T. E. Whall, Appl. Phys. Lett. 74, 579 (1999).

${ }^{6}$ Y. Guldner, J. M. Berroir, J. P. Vieren, M. Voos, I. Sagnes, P. A. Badoz, P. Warren, and D. Dutartre, Phys. Rev. B 48, 12312 (1993).

${ }^{7}$ C. J. Emeleus, M. A. Sadeghzadeh, P. J. Phillips, E. H. C. Parker, T. E. Whall, M. Pepper, and A. G. R. Evans, Appl. Phys. Lett. 70, 1870 (1997).

${ }^{8}$ T. Tesuka, T. Hatakeyama, S. Imai, N. Sugiyama, and A. Kurobe, Semicond. Sci. Technol. 13, 1477 (1988).

${ }^{9}$ M. A. Sadeghzadeh, O. A. Mironov, C. J. Emeleus, C. P. Parry, P. J. Phillips, E. H. C. Parker, and T. E. Whall, Acta Phys. Pol. A 94, 503 (1998), Proceedings of XXVII Intern. School on Physics of Semiconducting Compounds, Jaszowiec (1998).

${ }^{10}$ F. Schäffler, Semicond. Sci. Technol. 12, 1515 (1997).

${ }^{11}$ G. Hionis, and G. P. Triberis, Superlattices Microstruct. 22, 285 (1997). 